\title{
Detection of Calcium-induced morphological changes on RBCs by digital holographic microscopy and blinking optical tweezers
}

\author{
Vahideh Farzam Rad ${ }^{*}$, Rahim Tavakkoli ${ }^{*}$, Ali-Reza Moradi ${ }^{*}+, * ;$, Arun Anand ${ }^{\#}$, and Bahram Javidi ${ }^{\S}$ \\ * Department of Physics, University of Zanjan, 45195-313, Zanjan, Iran \\ ${ }^{\dagger}$ Optics Research Center, Institute for Advanced Studies in Basic Sciences, 45137-66731, Zanjan, Iran \\ * Department of Physics, Bilkent University, 06800, Cankaya, Ankara, Turkey \\ \# Applied Physics Department, MS University of Baroda, 390001,Vadodara, India \\ $\S$ Department of Electrical and Computer Engineering, University of Connecticut, Storrs, CT 06269-2157, USA
}

\begin{abstract}
Ca}^{+2}$ level in the circulating red blood cells (RBCs) takes part not only in controlling biophysical properties, but also affects the membrane composition, and its morphological and rheological properties. Excessive accumulation of $\mathrm{Ca}^{2+}$ within the cells is associated with a number of important pathological diseases. In this paper, by the use of digital holographic microscopy (DHM), we quantitatively analyzed the volumetric behavior of RBC membrane under influence of excess Calcium ions. DHM in a transmission mode is an effective tool for quantitative visualization of phase objects. By deriving the associated phase changes $3 \mathrm{D}$ information on the morphology variation of the cells at arbitrary time scales is obtained. Individual cells are immobilized by the use of optical tweezers and are monitored live with DHM system, while the concentration of $\mathrm{Ca}^{2+}$ ions in the buffer is changed simultaneously. We utilized blinking optical tweezers, by inserting an optical chopper to modulate intensity of the trapping laser beam. Blinking optical tweezers, while keeping the cell trapped during the experiments, ensures of minimizing the photo-damage of trapping laser beam on the cell. Our experimental results are in agreement with previous biological studies and predictions, and experimental observations of living RBCs under $\mathrm{Ca}^{2+}$ influence.
\end{abstract}

\section{INTRODUCTION}

It is obvious that for many cases DHM technique may be applied when the sample to be imaged and analyzed is immobilized. On the other hand, there is a large class of micron-sized important objects, in particular living cells and aerosol particles, which require keeping in a liquid or gaseous environment for their normal functionality [1]. In such size ranges, a long time DHM imaging and tracking the samples without combining it with an immobilization technique will be avoided by Brownian motion, motility of living cells, and fluxes in the medium. Using a pipette or other known physical or chemical methods for living cell immobilization on planar surfaces may induce undesirable or noisome changes in biological samples. Chemical immobilization can change the chemical environment of the living cell and may yield other unknown effects.
An elegant approach for immobilization of micron-sized samples in suspension is using the concept of an optical trap or optical tweezers. The optical trap (OT) uses radiation pressure of a laser beam, that is focused through a high numerical aperture microscope objective or tapered optical fibers, to trap and manipulates micron sized particles [2-4]. Optical trapping up to a high extent is nondestructive, non-invasive, and sterile, and requires little or no sample preparation. With optical traps samples can be kept and studied for up to several hours making the technique very useful for a variety of biological and medical studies including cell living processes. They have been used to apply and to measure forces in the $\mathrm{pN}$ range, to trap dielectric microspheres, viruses and bacteria, living cells, organelles and small metal particles, and to stretch and alter larger structures such as cell membranes and vesicles [5-8].

The establishment of both DHM and OT on a microscope makes combining the two techniques straightforward. Several configurations that are distinct by the propagation directions of the OT and DHM beams may be proposed for combined DHM-OT systems to 3D quantitative visualization of 3D optically trapped structures [9-11]. In in-line DHM

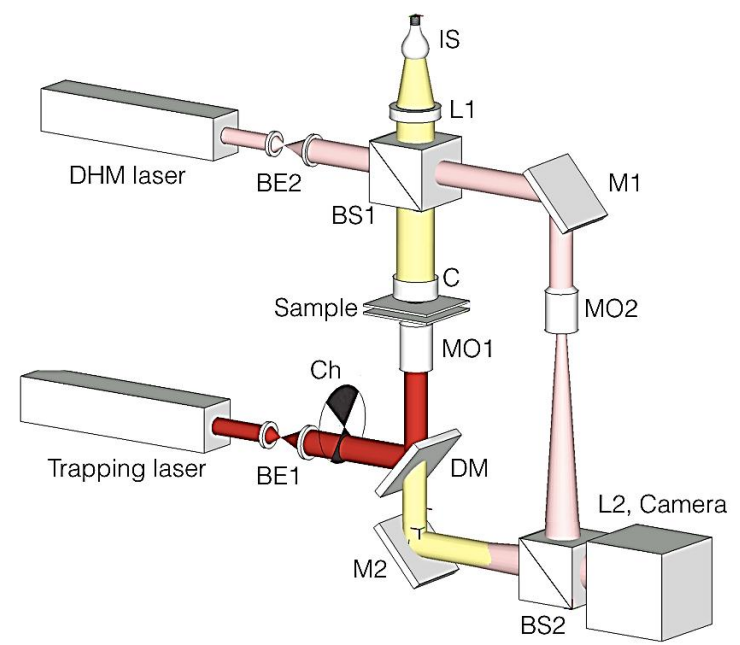

Fig. 1. Off-axis DHM integrated with blinking OT setup. L: lens, M: mirror, C: condenser, DM: dichroic mirror, MO: microscope objective, BE: beam expander, BS: beam splitter, Ch: optical chopper. 
configuration the coherent superposition of the laser light scattered from the trapped object with the un-diffracted portion of the laser beam builds the digital hologram. However, in-line DHM suffers of superimposing of the bright reconstructed wave avoiding clear $3 \mathrm{D}$ reconstruction of the samples [12].

In this paper, we utilize off-axis DHM-OT configuration for quantitative detection of the RBC morphology changes under exposure of $\mathrm{Ca}^{+2}$ elevation in the medium [13]. We have used an optical chopper to cut the trapping laser beam periodically and building a blinking OT. Blinking OT minimizes the photo-damage of laser beam on the cell while keeping the cell trapped during the experiment.

\section{EXPERIMENTAL SETUP AND RESULTS}

Fig. 1 shows the scheme of our setup. Illumination source (IS), collecting lens (L1), condenser (C), microscope objective (MO1), tube lens (L2) and imaging Camera is a conventional inverted microscope. The trapping laser is expanded by BE1 and after modulation by the optical chopper is sent to the microscope objective MO1 by the dichroic mirror DM. MO1 focuses the trapping beam in the sample and builds the traps. In this setup the DHM laser beam after expanding by BE2 by the beam splitter BS1 is divided into two; The beam going through mirror M1 is the reference beam which is sent into the camera through BS2. The object beam goes through the condenser onto the trapped object and the scattered beam from the object will go through MO1, M2 and BS2 to interfere with the reference beam to construct the digital hologram. Optical trapping is usually performed using high numerical aperture (NA) objectives which has short working distance (WD). Therefore, the main benefit of this scheme is that using low WD objectives is possible. However, since OT and DHM have a common propagation direction through the same objective, the objective can be optimized either for holographic imaging or for optical trapping. Adjustment of the microscope objective for DHM may cause dislodging of the trapped objects. The trapping laser is a collimated polarized laser (DPSS, 85- GSS- 309, Melles Griot, $532 \mathrm{~nm}$ ). The laser beam in the setup of Fig. 1, after intensity modulation by the optical chopper passes through the microscope objective (UPLFLN, 100X, NA=1.3, Olympus). DHM beam is a He-Ne laser (MEOS, $632.8 \mathrm{~nm}, 5 \mathrm{~mW}$ ) that goes through the trapped object and interferes with the reference beam on the recording camera (DCC1545M, Thorlabs, 8 bit dynamic range, $5.2 \mu \mathrm{m}$ pixel pitch).

We have used the combined setup to trap RBCs and study their morphology. We trapped a $\mathrm{RBC}$ within a rectangular capillary tube (VitroCom) that is connected from both ends to an infusion micro-pump. The concentration of the $\mathrm{Ca}^{+2}$ around the trapped cell can be adjusted by injecting $\mathrm{CaCl}_{2}$ from one end of the capillary and withdrawing $\mathrm{NaCl}$ ( $\mathrm{RBC}$ 's normal buffer) from the other end. This is performed by a push-pull procedure provided by the micro-pump, which also ensures of keeping the volume of the sample constant. We trapped RB Cs while increasing the concentration of $\mathrm{Ca}^{2+}$ ions in the
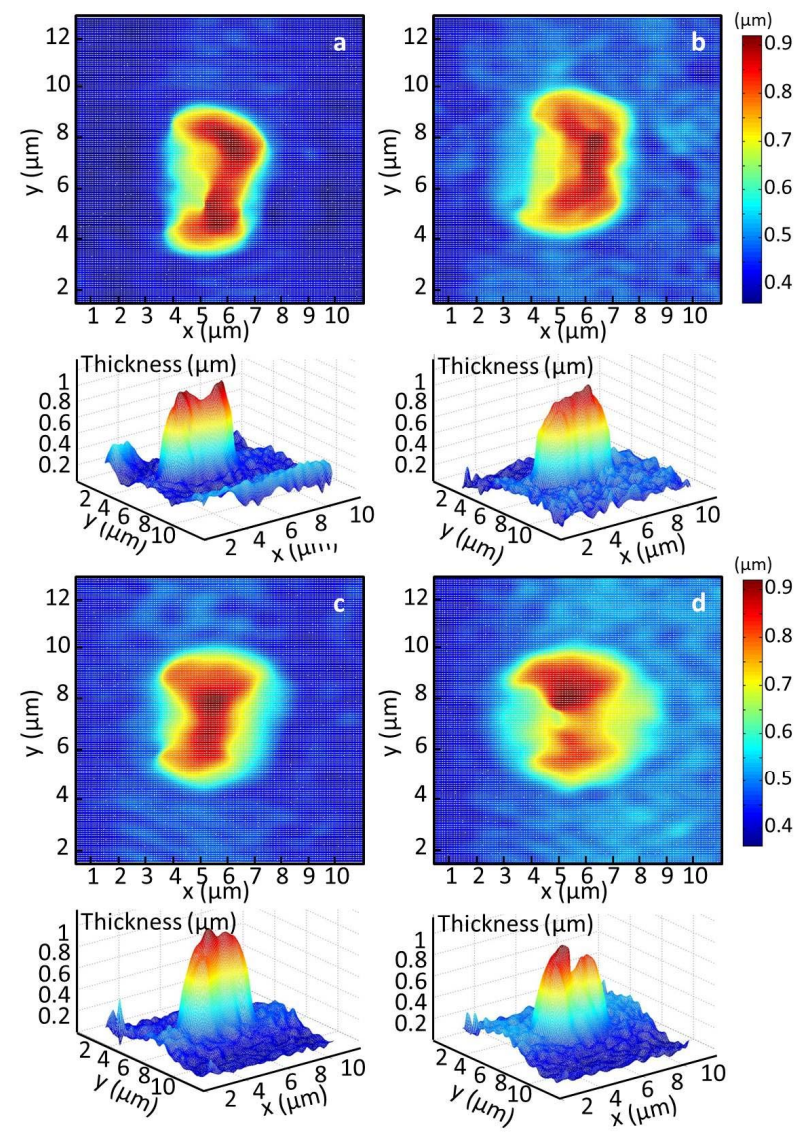

Fig. 2. $2 \mathrm{D}$ and $3 \mathrm{D}$ reconstructed images of a trapped $\mathrm{RBC}$ using the off-axis DHM-OT setup. (a) the cell at the beginning of experiment in its normal buffer, and in $\mathrm{CaCl}_{2}$ concentrations of approximately (b) $1.3 \mathrm{mM}$, (c) 2.6 $\mathrm{mM}$, and (d) $3.9 \mathrm{mM}$ in the solution.

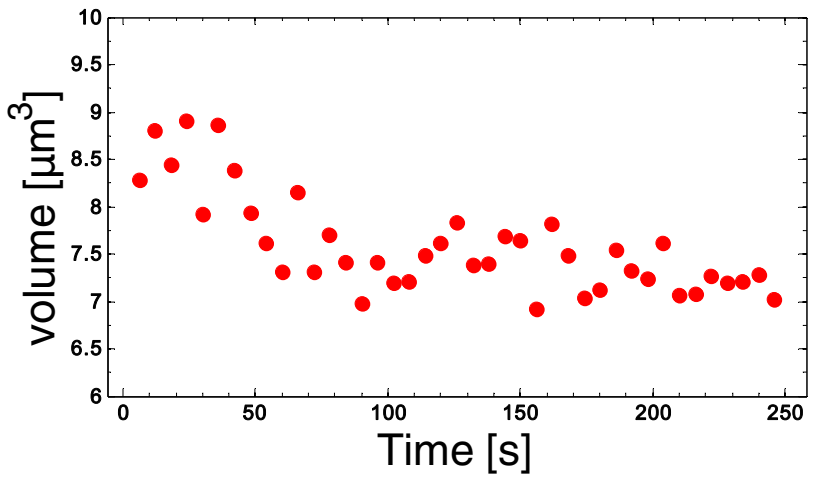

Fig. 3. Variations of average volume of sets of RBCs vs. time after starting the infusion of $\mathrm{CaCl}_{2}$.

medium and acquired digital holograms from the cells at time intervals of $0.2 \mathrm{~s}$. We utilized the angular spectrum propagation approach in scalar Fresnel-Kirchhoff diffraction theory for numerical reconstruction of the recorded holograms [14]. Within the reconstruction process, the recorded holograms are numerically propagated to the reconstruction plane, from which the phase and intensity of the reconstructed wavefront can be computed. The reconstructed phase pattern 
is unwrapped by Goldstein's branch-cut method to convert it into continuous-phase distribution leading to quantitative phase contrast image of the sample. From the reconstructed phase or intensity pattern the area covered by a RBC can be calculated. Assuming negligible changes for refractive index, the thickness at an arbitrary point on the trapped RBC can also be calculated and hence the volume can be obtained.

Figs. 2 (a)-(d) show the 2D and 3D reconstructed images of the $\mathrm{RBC}$, corresponding to the beginning of experiment, that the cell is in its normal buffer, and to three different $\mathrm{CaCl}_{2}$ concentrations of approximately $1.3 \mathrm{mM}, 2.6 \mathrm{mM}$, and 3.9 $\mathrm{mM}$ in the solution, respectively. Elevation of $\mathrm{Ca}^{2+}$ concentration induces morphological changes to the trapped cell, which can be calculated in terms of cell volume and the roughness of the cell surface. This information can be obtained from reconstruction of the digital holograms. In dynamic phenomena the phases in various states are computed individually, the phase differences provide information on the changes occurring to the object. Figure 3 shows the variations of average volume of sets of RBCs after infusion of $\mathrm{CaCl}_{2}$ into the cell chamber, hence, in presence of different concentrations of $\mathrm{Ca}^{2+}$ ions. The results are in agreement with previous studies. In our previous study we observed the morphology changes indirectly by monitoring the forward scattering light of the laser from the trapped cell and its dependence on the size and drag coefficient of the cell [13]. Also the results are in agreement with earlier studies based on induced hemolysis measurement for various kinds of incubations of the cells with calcium. Calcium ions are known to cause aggregations reducing the lateral diffusion of lipid molecules and retarding the motion of alkyl chains. This leads to the reduction in the transverse movement of molecules and ions across the aggregates [15]. $\mathrm{Ca}^{2+}$ can cause local aggregation of phospholipids bilayers in the membrane, but it causes an overall decrease in the membrane rigidity resulting in greater hemolysis [16]. In short, our observation can be interpreted as a result of increased localized aggregation but decreased overall rigidity of phospholipid bilayers in the membrane of RBCs.

\section{REFERENCES}

[1] M, T, and J. Alper, Challenges in Characterizing Small Particles:: Exploring Particles from the Nano-to Microscales, National Academies Press, USA, 2012.

[2] A. Ashkin and J. Dziedzic, "Stability of optical levitation by radiation pressure," Appl. Phys. Lett., vol. 24, pp. 586-588, 1974.

[3] K. C. Neuman and S. M. Block, "Optical trapping," Rev. Sci. Instrum., vol. 75, pp. 2787-2809, 2004.

[4] S. K. Mohanty and M. W. Berns, "Manipulating of mammalian cells using single-fiber optical microbeam," J. Biomed. Opt., vol. 13, pp. 054049, 2008.

[5] A. Rohrbach and E. H. K. Stelzer, "Trapping forces, force constants, and potential depths for dielectrical spheres in the presence of spherical aberrations," Appl. Opt., vol. 41, pp. 2494-2507, 2002.

[6] A. Ashkin and J. M. Dziedzic, "Optical trapping and manipulation of viruses and bacteria," Science, vol. 235, pp. 1517-1520, 1987.

[7] A. Ashkin, J. M. Dziedzic, and T. Yamane, "Optical trapping and manipulation of single cells using infrared-laser beam," Nature, vol. 330, pp. 769-771, 1987.

[8] A. Ashkin and J. M. Dziedzic, "Internal cell manipulation using infrared-laser traps," Proc. Nat. Acad. Sci., vol. 86, pp. 7914-7918, 1989.
[9] S. H. Lee and D. Grier, "Holographic microscopy of holographically trapped three-dimensional structures," Opt. Express, vol. 15, pp. 15051512, 2007.

[10] M. Danesh Panah, S. Zwick, F. Schaal, M. Warber, B. Javidi, and W. Osten, "3D holographic imaging and trapping for non-invasive cell identification and tracking," IEEE J. Dis. Tech., vol. 6, pp. 490-499, 2010.

[11] L. Selvaggi, E. Ferrari, A. R. Moradi, S. Santucci, and D. Cojoc, "Optimized multi-view imaging improves the observation of optically manipulated non-spherical particles," J. Opt., vol. 12, pp. 035303, 2010.

[12] M. K. Kim, "Principle and techniques of digital holographic microscopy," SPIE Rev., vol. 1(1), 2010.

[13] A. R. Moradi, M. K. Ali, M. Daneshpanah, A. Ananad, B. Javidi, "Detection of Calcium-induced morphological changes of living cells using optical traps' IEEE Photonics J., vol. 2, pp.775-783, 2010.

[14] A. Anand, V. K. Chhaniwal, and B. Javidi, "Real time digital holographic microscopy for phase contrast 3D-imaging of dynamic phenomena," J. Disp. Tech., vol. 6, pp. 500-505, 2010.

[15] S. Ohnishi and T. Ito, "Calcium-induced phase separation in phosphatidylserine-phosphatidylcholine membranes," Biochem., vol. 13, pp. 881-887, 1974.

[16] M. K. Ali and S. Tayyab, "Calcium-induced bilirubin-dependent hemolysis of human erythrocytes," Biochem. Biophys. Acta, vol. 1326, pp. 124-130, 1997. 